\title{
Impact of anaesthetic management on postoperative outcome of patients undergoing cytoreduction surgery with hyperthermic intraperitoneal chemotherapy
}

\section{Hamed ELGENDY, MD,PhD 1.2.3; Hanaa Nafady-Hego 4,5, Hanan Abd ElMoneim 6,7 , Talha}

YOUSSEF , MBBS 8 , Abdel Aziz Alzahrani ${ }^{9}$,

1 Department of Anesthesia, Assiut University Hospitals, Egypt \& 2 Anesthesia Dept., HAMAD Medical Corporation, Doha, Qatar \& 3 Clinical Ass. Prof. Weill Cornell Medicine Qatar.

${ }^{4}$ Faculty of Medicine- Assiut University, Microbiology and Immunology, Assiut, Egypt.

${ }^{5}$ Faculty of Medicine- Umm Alqura University, Haematology and Immunology, Makkah, Saudi Arabia.

${ }^{6}$ Faculty of Medicine- Minia University, Pathology, Minia, Egypt.

${ }^{7}$ Faculty of Medicine- Umm Alqura University, Pathology, Makkah, Saudi Arabia.

${ }^{8}$ Prince Mohammad Bin Abdul-Aziz Hospital- Ministry of National Guard, Internal Medicine, Al Madinah al-Munawarah, Saudi Arabia.

${ }^{9}$ King Abdullah Medical City, Surgery, Makkah, Saudi Arabia.

\section{Background and aims}

Cytoreductive surgery (CRS) with hyperthermic intraperitoneal chemotherapy (HIPEC) plays an important role in the management of peritoneal surface malignancy. These procedures are complex and stressful commonly leads to coagulation derangements and electrolytes disturbances.

Our aim is to analyze anaesthetic management of HIPEC patients and investigate its impact on patient's outcome.

Methods

We prospectively observed thirty-eight cases with abdominal malignancy underwent HIPEC, (2012-2015). Serial arterial blood gases sampling for electrolytes, blood sugar, coagulation control and maintenance of normovolemia.

\section{Results}

Majority of patients were female 23 (60.5\%) and ASA III, 23 (60. 5\%). Changes in haemodynamics, temperature, glucose, and lactate (Fig.1A-B). Our patients had overall postoperative mortality $42.1 \%$, (Fig.2). Univariate analyses of variables that can affect outcome after HIPEC demonstrated that low haemoglobin, haematocrite, tumour marker (CA19.9), low potassium, calcium, albumin, higher intra operative colloids transfusion ,postoperative activated partial thromboplastin time, bacterial and fungal infections were potentially risk factors affected patient's mortality. Multivariate analysis of those factors showed that lower preoperative calcium (hazard ratio $[\mathrm{HR}]=0.116 ; 95 \%$ confidence interval $[\mathrm{Cl}]=0.033-0.407 ; \mathrm{P}=0.001$ ), higher plasma transfusion $(\mathrm{HR}=1.004 ; 95 \% \mathrm{Cl}=1.001-1.003$;

$\mathrm{P}=0.012$ ) and higher postoperative bacterial infections (HR $=5.987 ; 95 \% \mathrm{Cl}=1.009-35.54 ; \mathrm{P}=0.014)$

were independent predictors of patient death, (Table 1).

\section{Conclusions}

Lower preoperative calcium, higher intra-operative plasma protein transfusion and postoperative bacterial infection were independent predictors of patient death after HIPEC. Balanced perioperative management of temperature, acidbase balance together with maintenance of normovolmic status are crucial in that critical procedure.

Fig. 1

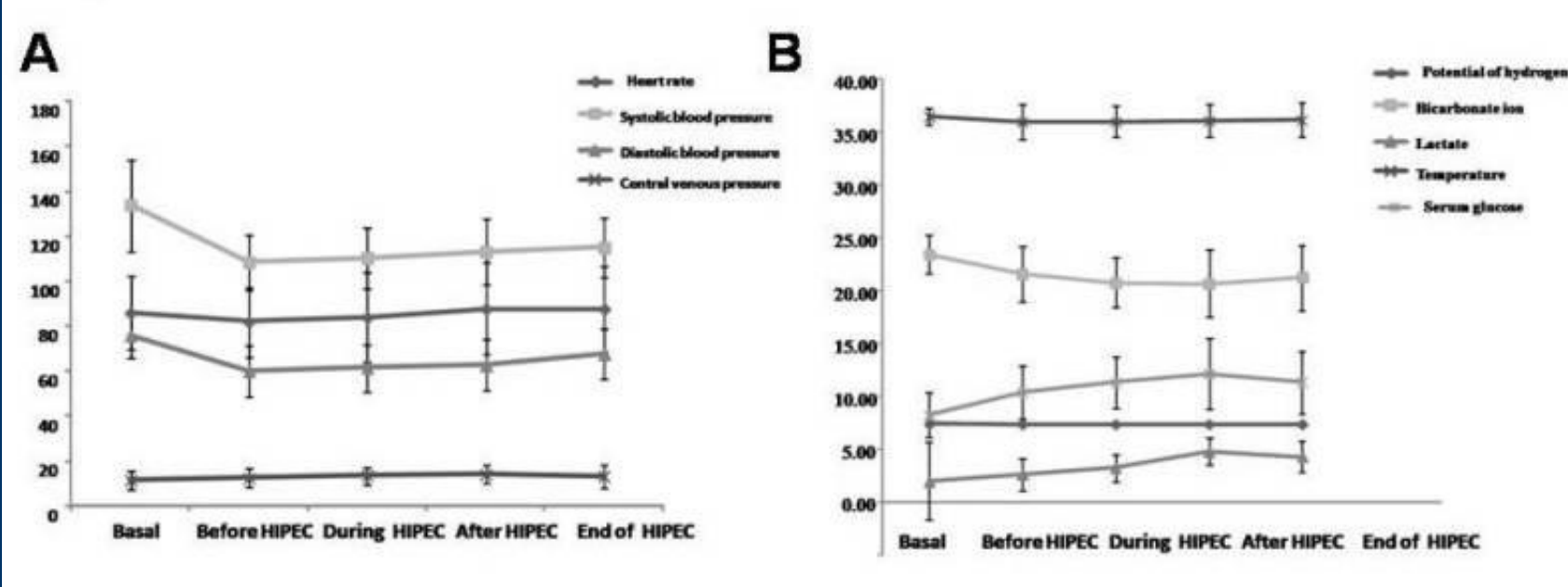

Fig.2

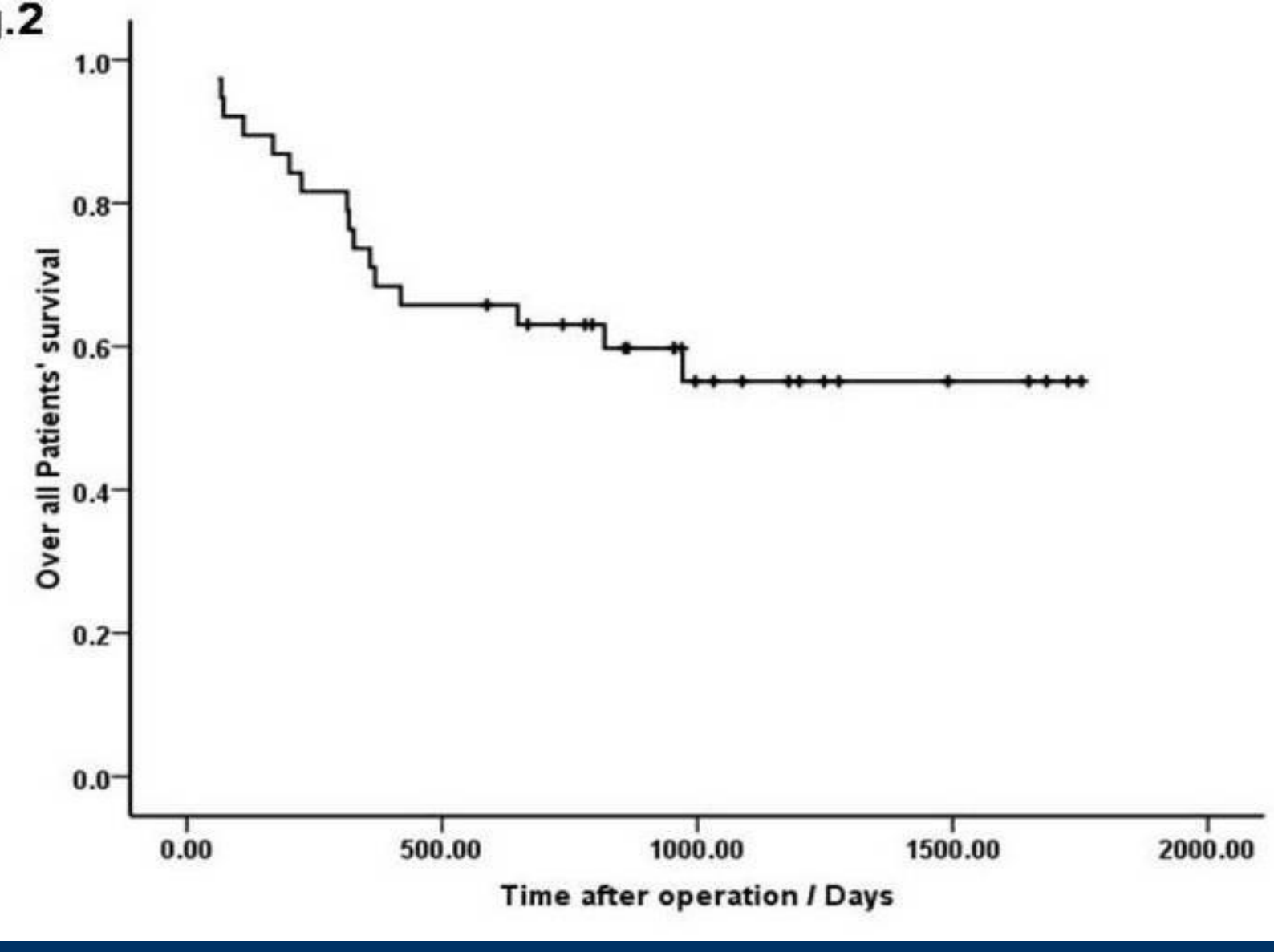

Table 1.Univarialle- and multivariable analysis with respect to overall survival in patients after HIIEC operation

\begin{tabular}{|c|c|c|c|c|c|c|}
\hline \multirow[b]{2}{*}{ Variable } & \multicolumn{3}{|c|}{ Univariable cox model } & \multicolumn{3}{|c|}{ Multivariable cox model } \\
\hline & HR & $(95 \% \mathrm{Cl})$ & P Value & $\mathrm{HR}$ & $(95 \% \mathrm{Cl})$ & P Value \\
\hline Preoperative $\mathrm{MCH}$ & 1.094 & $(1.009-1.186)$ & 0.03 & & & \\
\hline Preoperative Haemoglobulin & 0.693 & $(0.486-0.988)$ & 0.043 & & & \\
\hline Preoperative HCT & 0.864 & $(0.763-0.978)$ & 0.021 & & & \\
\hline Preoperative CA19.9 & 1.03 & $(1.008-1.053)$ & 0.008 & & & \\
\hline Preoperative Potassium & 0.147 & $(0.039-0.559)$ & 0.005 & & & \\
\hline Preoperative Calcium & 0.246 & $(0.096-0.628)$ & 0.003 & 0.116 & $(0.033-0.407)$ & 0.001 \\
\hline Preoperative albumin & 0.3 & $(0.093-0.965)$ & 0.043 & & & \\
\hline $\begin{array}{l}\text { Preoperative Total protein } \\
\text { Intra operative Plasma protein }\end{array}$ & 0.657 & $(0.463-0.965)$ & 0.018 & & & \\
\hline $\begin{array}{l}\text { Intra operalıve Plasma protem } \\
\text { transfision }\end{array}$ & 1.001 & $(1-1.002)$ & 0.049 & 1.004 & $(1.001-1.003)$ & 0.012 \\
\hline $\begin{array}{l}\text { Intra operative Colloids transfusion } \\
\text { Postoperative APTT with }\end{array}$ & 1.002 & $(1.001-1.003)$ & 0.002 & & & \\
\hline Heparin/sec & 1.036 & $(1.003-1.071)$ & 0.035 & & & \\
\hline Postoperative Bacterial infection & 6.494 & $(1.471-28.673)$ & 0.014 & 5.987 & $(1.009-35.54)$ & 0.049 \\
\hline Postoperative Fungal infection & 3.488 & $(1.185-10.265)$ & 0.023 & & & \\
\hline
\end{tabular}

